\title{
MicroRNA-183 Suppresses Neuropathic Pain and Expression of AMPA Receptors by Targeting mTOR/VEGF Signaling Pathway
}

\author{
Xiaojuan Xie Ligang Ma $^{\mathrm{b}}$ Kai Xic ${ }^{\mathrm{a}}$ Dongmei Fan ${ }^{\mathrm{d}}$ Wei Zhang ${ }^{\mathrm{a}}$
}

\begin{abstract}
aDepartment of Anesthesia, the First Affiliated Hospital of Zhengzhou University, Zhengzhou, ${ }^{b}$ Department of Anesthesia, the First Affiliated Hospital of Henan Science-Technology University (New District Hospital), Luoyang, 'Department of ENT, the First Affiliated Hospital of Henan ScienceTechnology University (New District Hospital), Luoyang, dDepartment of Obstetrics and Gynecology, the First Affiliated Hospital of Henan Science-Technology University, Luoyang, China
\end{abstract}

\section{Key Words}

Neuropathic pain - miR-183 - mTOR/VEGF Signaling Pathway - AMPA receptors • Chronic compress injury model

\begin{abstract}
Background: Neuropathic pain is a type of chronic pain that results from dysfunctions of the somatosensory nerve system. This study was aimed to investigate the effect of mTOR/VEGF signaling pathway on neuropathic pain and the regulation mechanisms of miR-183 on AMPA Receptors through mTOR/VEGF signaling pathway. Methods: Chronic compress injury (CCI) model was constructed in the current study, we used paw withdrawal mechanic threshold (PWMT) and paw withdrawal thermal latency (PWTL) to observe mTOR and VEGF receptors. Dual luciferase analysis, western blot and qRT-PCR were also applied to complete this experiment. Results: It was observed that the inhibition of mTOR and VEGF receptors could significantly relieve neuropathic pain in the CCI model. Moreover mTOR was confirmed as the direct target of miR-183. Furthermore, miR-183 could modulate VEGF through regulating mTOR expressions. We also found the expressions of AMPA receptors (i.e. GluR1 and GluR2), located in the downstream of mTOR/VEGF signaling pathway, were significantly upregulated when miR-183 was downregulated or when the mTOR/VEGF signaling pathway was activated. Conclusion: The inhibition of mTOR or VEGF receptors can significantly relieve neuropathic pain, and the upregulation of miR-183 can suppress AMPA receptors by inhibiting mTOR/ VEGF pathway.
\end{abstract}




\section{Cellular Physiology Cell Physiol Biochem 2017;41:181-192

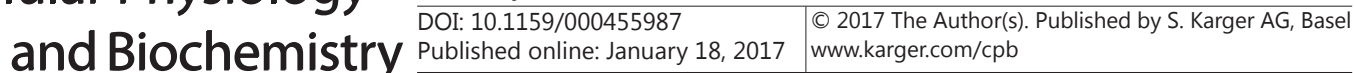 \\ Xie et al.: MicroRNA-183 Targets mTOR/VEGF Signaling Pathway in Neuropathic Pain}

\section{Introduction}

Neuropathic pain is a type of chronic pain that occurs when nerves in somatosensory nerve system were dysfunctional, injured or damaged [1]. It is estimated that more than $20 \%$ of cancer pains, a result of tumor metastasis or side effect of chemotherapy, are of neuropathic origin [2]. It is also reported that around $7 \%$ of the population in France suffered from chronic pain with neuropathic characteristics [3]. Although the prevalence is very high, the treatments of neuropathic pain, analgesics and neurosurgical interventions such as electrical nerve stimulation, have disappointing efficacy and undesirable risk benefit ratio [4]. Despite the fact that decades were spent to clarify the specific mechanisms of neuropathic pain and relieve the pain of the patients, the study is still in its infancy.

MicroRNAs (miRs) are a group of small non-coding RNAs that play a critical role in gene regulation $[5,6]$. By binding to protein-coding mRNA with complementary sequences, miRNAs can regulate gene expression post-transcriptionally [7]. MiR-183 family is a group of special miRNAs that are abundant in nerve system [8]. Altered expression of miR-183 family in nerve system may modify the process of neuropathic pain. Researchers have observed that the expression of miR-183 was significantly down-regulated in dorsal root ganglion after spinal nerve ligation, which is correlated with mechanical allodynia [8, 9]. However, the downstream target of miR-183 has not been identified.

Mammalian target of rapamycin (mTOR) is a conserved serine/threonine kinase that plays an important part in physiological and pathological processes [10]. MTOR has been considered as a key player in cellular adaptive responses to energy, nutrient, growth factor levels and stress stimuli [11]. As proved by previous study, the activated form of mTOR is up-regulated in peripheral nerve system and spinal cord in pain models [12]. Systematic inhibition of mTOR alleviates the mechanical hypersensitivity associated with inflammation and neuropathy without damaging sensory [13].

Vascular endothelial growth factor (VEGF) is a crucial regulator in angiogenesis, and five distinct genes are encoded in human, i.e. VEGF-A, VEGF-B, VEGF-C, VEGF-D and PLGF (placenta growth factor) [14]. Previous researches have indicated that modified VEGF-VEGFR (VEGF receptor) signaling pathway has an impact on the development of many diseases, including carcinoma and inflammatory diseases [15-17]. In previous studies, researchers observed that tumor-derived VEGF-A, PLGF-2, and VEGF-B could augment pain sensitivity by activating VEGFR1 in sensory neurons, while blockade of VEGFR1 attenuated cancer pain in the same model [18]. Meanwhile, mTOR was found to be able to regulate VEGF signaling pathway via dysregulation of HIF [19-21]. Thus we suspected that mTOR/VEGF signaling pathway may play a part in neuropathic pain.

The $\alpha$-amino-3-hydroxy-5-methyl-4-isoxazolepropionic acid (AMPA) receptors are ionotropic transmembrane receptors for glutamate [22]. Most AMPA receptors are heterotetrameric complexes, consisting of glutamate receptor (GluR) 2 and GluR1, GluR3 or GluR4 [23]. As suggested by previous research, GluR2 trafficking in the periaqueductal grey could modulate pain and potent analgesic effects were observed in rat model by inhibiting GluR2 endocytosis [24]. Besides, it is also observed that AMPA receptor subunits were upregulated in dorsal root ganglion following nerve injury [25].

In this study, we investigated the potential target of miR-183 in the regulation of neuropathic pain. We aimed to gain further understanding of the pathological mechanism of neuropathic pain and search for novel targets of therapy.

\section{Materials and Methods}

Animals and surgery

Thirty-five clean adult male Sprague-Dawley (SD) rats (280 - 400 g) were provided by the First Affiliated Hospital of Zhengzhou University. They were fed separately in cages with an indoor-constant temperature of $25^{\circ} \mathrm{C}$. The SD rats were then randomly divided into the following 7 groups $(\mathrm{n}=5 \mathrm{in}$ each): 


\section{Cellular Physiology Cell Physiol Biochem 2017;41:181-192

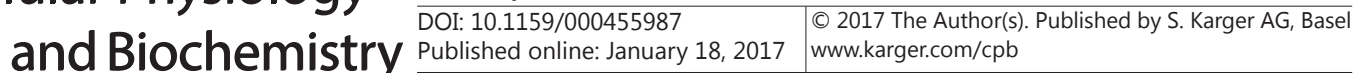 \\ Xie et al.: MicroRNA-183 Targets mTOR/VEGF Signaling Pathway in Neuropathic Pain}

(1) chronic compress injury (CCI) model: separation and ligation of the medial side of the left sciatic nerve; (2) sham group: separation and exposure of the left sciatic nerve with coiling, yet no ligation; (3) rapamycin group: sheath built-in tube, separation and ligation of the medial side of the left sciatic nerve and injection of rapamycin (Beyotime Shanghai) within sheath 4 hours after surgery for consecutive 3 days; (4) antiVEGF group: sheath built-in tube, separation and ligation of the medial side of the left sciatic nerve and injection of anti-VEGF (R\&D AF564) within sheath 4 hours after surgery for consecutive 3 days; (5) DMSO group: sheath built-in tube, separation and ligation of the medial side of the left sciatic nerve and injection of DMSO (solvent of rapamycin) (Invitrogen); (6) NS group: sheath built-in tube, separation and ligation of the medial side of the left sciatic nerve and injection of NS (solvent of anti-VEGF) within sheath 4 hours after surgery for consecutive 3 days; (7) control group: normally fed rats with no treatments. As for the surgery, the SD rats were given modified sheath built-in tube in the waist until after peritoneal injection with $10 \%$ chloral hydrate $(350 \mathrm{mg} / \mathrm{kg}$ ). The procedure of sheath built-in tube surgery all fully complied with Takei et al. [26]. Furthermore, CCI animal model was established in light of Bennett-Xie et al. [27]. Briefly, the skin of mid-thigh was incised in the periphery of thighbone and inter-muscular fascia was bluntly dissected along the muscle clearance, enabling the sciatic nerve trunk exposed. Four ligatures (4-0 chromic gut) were tied (interval: $1.0 \mathrm{~mm}$ ) proximal to the sciatic trifurcation so that calf muscle trembled slightly and blood supply of epineurium would not be affected. The experiment has been approved by the First Affiliated Hospital of Zhengzhou University experimental animal committee.

Testing of paw withdrawal thermal latency (PWTL) and paw withdrawal mechanic threshold (PWMT)

PWTL and PWMT of rats' left hind paws were assessed with application of 7370 Plantar Test (Ugo Basile, Italy) and 2390 Electronic von-Frey Anesthesiometer (IITC Life Science, USA) [27]. For the evaluation of PWTL, light source was pointed to rats' paws below the pelma and the positions were the same for each testing. When the rats responded with leg rising because of self-reported pain, the recorded time was considered PWTL for this testing (accurate to $0.1 \mathrm{~s}$ ). All rats were measured three times, and the time interval lasts for 5 minutes at least. As for another appraisal of PWMT, the light source was replaced with a probe touching the pelma of rats. PWTL and PWMT were tested for all groups of rats 1 day before modeling as well as on the $1^{\text {st }}, 3^{\text {rd }}, 5^{\text {th }}$ and $7^{\text {th }}$ day after modeling.

\section{Sampling and specimen preparation}

All rats were sacrificed on $8^{\text {th }}$ day after modeling, and the spinal cord (L4-L6) was taken. One part was transferred into liquid nitrogen tank $\left(-80^{\circ} \mathrm{C}\right)$ for the following detection of the expression of miR-183, and related mRNAs and proteins in superficial laminae of spinal dorsal horn. The other part was fixed in $4 \%$ formic acid polymer for 12 hours and then kept in PBS with sodium azide. After dehydrated with gradient ethanol, transparentized with butanol and embedded with paraffin, serial section cutting was performed with rotary microtome (Shandon, England) and slice thickness was guaranteed as $5 \mu \mathrm{m}$. Subsequently, the slices were baked in slide warmers for 4 hours and maintained in $37^{\circ} \mathrm{C}$ overnight.

\section{Determination of expressions of miR-183, mTOR and VEGF}

We extracted the total RNA of spinal cord following miRVana total RNA isolation kit (Ambion) and measured the amount of RNA with Nanodrop (Thermo Scientific, USA). The mRNA expressions were calculated in accordance with a previous protocol [28]. Briefly, total RNA was reversely transcribed to cDNAs [Archiving Kit (Life Tech, USA)], which were then amplified [Power SyBr Green PCR Master Mix (Life Tech, USA)]. The primer sequences for miR-183, mTOR and VEGF were shown as Table 1. Ultimately, we obtained the amplified signals through 7300HT fast real-time System (Applied Biosystem, USA) and normalized mRNA expressions with GAPDH intensity.

\section{Hematein eosin (HE) staining}

Paraffin sections with intact spinal cords were dewaxed in thermostat $\left(57^{\circ} \mathrm{C}\right)$ for $30 \mathrm{~min}$ and then cooled for $20 \mathrm{~min}$. After dewaxing and rehydration of the slices with dimethylbenzene and alcohol of different concentrations, the slices were immersed into hematoxylin for $2 \mathrm{~min}$ and washed with distilled water. Then $1 \%$ hydrochloric acid alcohol was used for 2 seconds, followed with running water wash. After that, eosin was used for $2 \mathrm{~min}$ for the staining of cytoplasm. Finally, the slices were naturally dried, sealed with neutral resins and photographed. 


\section{Cellular Physiology Cell Physiol Biochem 2017;41:181-192 and BiOChemistry Published \begin{tabular}{l|l} 
DOI: 10.1159/000455987 & $\begin{array}{l}\text { C } 2017 \text { The Author(s). Published by S. Karger AG, Basel } \\
\text { www.karger.com/cpb }\end{array}$
\end{tabular} \\ Xie et al.: MicroRNA-183 Targets mTOR/VEGF Signaling Pathway in Neuropathic Pain}

Table 1. Primer pairs for quantitative real-time polymerase chain reaction (qRT-PCR). RT: reverse transcription

\begin{tabular}{|c|c|}
\hline Gene/miRNA & Primer sequences \\
\hline \multirow{2}{*}{ VEGF } & Forward: 5'-AGGAGGAGGGCAGAATCATCA-3' \\
\hline & Reverse: 5'-TCTCGATTGGATGGCAGTAGC-3' \\
\hline \multirow{2}{*}{ mTOR } & Forward: 5'-GCCCCACCCCCATAGCTTCTCTC-3' \\
\hline & Reverse: 5'-CAGGACTCAGGACACAACTAGCCC-3' \\
\hline \multirow{2}{*}{ GAPDH } & Forward: 5'-GAAGGTGAAGGTCGGAGTC-3' \\
\hline & Reverse: 5'-GAAGATGGTGATGGGATTTC-3' \\
\hline miR-RT & 5'-GCGAGCACAGAATTAATACGACTCACTATAGG(T)18VN-3' \\
\hline miR-183 & 5'-TATGGCACTGGTAGAATTCACT-3' \\
\hline
\end{tabular}

Immunohistochemistry with 3'-diaminobenzidine (DAB) staining and semi-quantitative analysis

Spinal cord sections in good shape were managed in turn with dewaxing, dimethylbenzene infiltration for three times, graded ethanol dehydration, antigen retrieval $\left(80{ }^{\circ} \mathrm{C}, 20 \mathrm{~min}\right), 3 \%$ hydrogen peroxide $(5 \mathrm{~min})$ and finally normal sheep serum closed (40 min). The primary antibodies (mouse anti-rat mTOR antibody, 1:500, Abcame 32028; anti-VEGF, 1:800, SantaCruz Biotechnology; mouse anti-GluR1, 1:50, SantaCruz 5550; mouse anti-GluR2, 1:3000, Chemion MAB39) were applied and co-incubated at $4{ }^{\circ} \mathrm{C}$ overnight firstly and then at room temperature for $20 \mathrm{~min}$. The secondary antibody labeled with Biotin (Dako) and the streptomycin avidin named with HRP (Horseradish Peroxidase) (Dako) were then applied and incubated for $30 \mathrm{~min}$ at room temperature. Staining was performed using 3'-diaminobenzidine (DAB) and slices were counterstained with hemalum. Phosphate balanced solution (PBS) instead of a primary antibody, was considered as a negative control (NC) and a known positive antibody was regarded as a positive control. The distribution of positive cells were observed by optical microscope (Abcam, USA), and record the total number of positive cells of the left side of the spinal dorsal horn of shallow under high power field of vision $(400 \times)$.

\section{Cell culture and differentiation of PC12 cells}

PC12 cells (pheochromocytoma cell lines of rats) were purchased from the fundamental research center, Union Medical College, Chinese Academy of Medical Sciences. The thawed PC12 cells were centrifuged at $1000 \mathrm{rpm}$ for $5 \mathrm{~min}$ and the resultant supernatant was immigrated into 6-8 $\mathrm{ml}$ complete medium for static cultivation $\left(37^{\circ} \mathrm{C}, 5 \% \mathrm{CO}_{2}\right)$ during 3-4 days. Cell passage would proceed when culture media changed from fuchsia to yellow and 70\%-80\% visible cells under the microscope began to fuse. After addition of 50$100 \mathrm{ng} / \mathrm{ml}$ nerve growth factor (NGF) to PC12 cells (density: $5 \times 10^{5} / \mathrm{ml}$ ), cell morphology was observed under the inverted microscope. Twenty-four hours later, cells were adherent to the cells and axons were generated. With further culture of 7-10 days, 90\% cells grew by adherence and formed like neuron cells. The above cells were utilized for experiments in vitro.

Inhibition of miR-183 in PC12 cells with technology of Locked Nucleic Acid (LNA)

We managed the sequence for suppressing expressions of miR-183 as $+\mathrm{A}+\mathrm{G}+\mathrm{T}+\mathrm{G}+\mathrm{AATTCTACCAGTG}+\mathrm{C}+\mathrm{C}+\mathrm{A}+\mathrm{T}+\mathrm{A}$ with LNA (Exiqon), and that for negative control as $+\mathrm{C}+\mathrm{A}+\mathrm{T}+\mathrm{T}+\mathrm{AATGTCGGACAAC}+\mathrm{T}+\mathrm{C}+\mathrm{A}+\mathrm{A}+\mathrm{T}$. The symbol of ' + ' in the above sentences was representative of locked base. The process of LNA oligonucleotides' transfection into PC12 cells was carried out with help of Lipofectamine 2000 reagent (Invitrogen). All the procedures were strictly in accordance with manufacturer's instructions.

\section{Luciferase assay of miR-183 with mTOR}

The wide-type and mutant-type of ITOR 3' untranslated regions (UTR) luciferase reporter vectors (50 ng) were constructed. Either $50 \mathrm{nM}$ LNA inhibitors (Exiqon) or $50 \mathrm{nM}$ pre-miRNAs (Ambion) were, along with the established wild/mutant-type luciferase reporter vectors, transfected into PC12 cells via Lipofectamine 2000 (Invitrogen). Luciferase activity was figured out with the Dual-Luciferase Reporter Assay System (Promega) when transfection lasted for at least $48 \mathrm{~h}$. We also constructed reported vectors where miRNA-183 seed regions were mutated by virtue of Quick Change II XL Site Directed Mutagenesis Kit (Agilent).

\section{KARGER}




\section{Cellular Physiology Cell Physiol Biochem 2017;41:181-192 \\ \begin{tabular}{ll|l} 
DOI: 10.1159/000455987 & $\begin{array}{l}\text { O } 2017 \text { The Author(s). Published by S. Karger AG, Basel } \\
\text { www.karger.com/cpb }\end{array}$ \\
and Biochemistry Published online: January 18, 2017 &
\end{tabular} \\ Xie et al.: MicroRNA-183 Targets mTOR/VEGF Signaling Pathway in Neuropathic Pain}

\section{Plasmid construction, lenti-virus production, and transfection}

Lenti-viral plasmids (Sunbio Medical Biotechnology) were transfected by ways of Lipofectamine 2000 [29]. Groups of VEGF siRNA and mTOR siRNA were PC12 cells transfected with si-VEGF and simTOR (GenePharma, China), whereas the control groups were cells transfected without any sequence. The aforementioned transfection processes were all executed by virtue of Lipofectamine 2000 (Invitrogen).

\section{Western blot analysis}

After the tissue was defrosted, RIPA cracking liquid with multiple inhibitors was added (every $20 \mathrm{mg}$ tissue with $200 \mu \mathrm{l}$ RIPA cracking liquid). Then ultrasonic apparatus was used until fully cracking on the ice, followed with centrifugation $\left(4{ }^{\circ} \mathrm{C}, 15000 \mathrm{r} / \mathrm{min}, 20 \mathrm{~min}\right)$. For the purpose of quantifying protein expressions, western blot was performed as described elsewhere [30]. In addition, antibodies for western blot assays were obtained as mTOR (Cell Signaling), VEGF (R\&D AF564), GluR1 (SantaCruz 55509), GluR2 (Chemion MAB39) and GAPDH (Ambion).

\section{Statistical analysis}

We performed all statistical analyses by SPSS 19.0 software. The numerical data (mean \pm SD) were measured by one-way analysis of variance (ANOVA) together with SNK-q post-test to evaluate the differences among the multiple groups. $P<0.05$ provided evidence for statistical significance.

\section{Results}

\section{PWMT and PWTL of rats' left hind}

Pre-operative PWMTs of all groups were fairly consistent $(P>0.05)$, and no pronounced distinctions were examined between control group and sham group in their capabilities to endure mechanical stimuli at every experimental point in time $(P<0.05)$ (Fig. 1A, Table 2). In contrast, among the CCI, NS, DMSO, rapamyclin or anti-VEGF groups, notable decrease

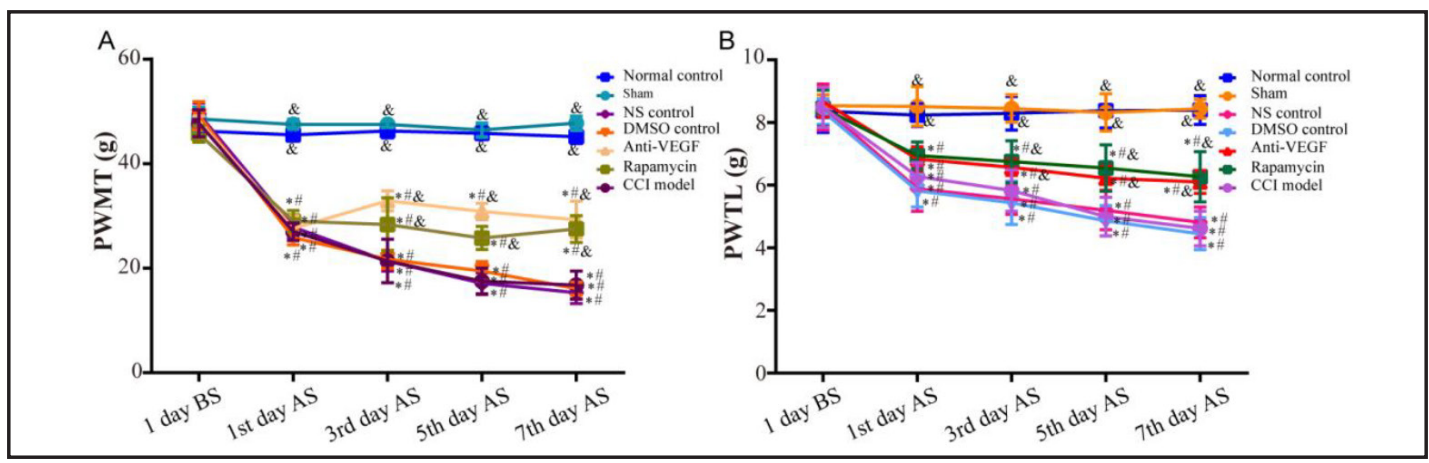

Fig. 1. PWMT (paw withdrawal mechanical threshold) and PWTL (paw withdrawal thermal latency) of all rats' posterior limbs. (A) PWMT; (B) PWTL. ${ }^{8}$ Comparisons with CCI group, $P<0.05$; ${ }^{*}$ Comparisons with normal control group, $P<0.05$; ${ }^{\#}$ Comparisons with 1 day BS, $P<0.05$.

Table 2. PWMT of rats' left posterior limbs ( $\mathrm{n}=25$, mean \pm SD, unit: g). PWMT: paw withdrawal mechanical threshold; SD: standard deviation; BS: before surgery; AS: after surgery; CCI: chronic constrictive injury; DMSO: dimethylsulfoxide; VEGF: vascular endothelial growth factor; NS: normal saline. ${ }^{\triangle}$ Comparisons with CCI group, $P<0.05$; "Comparisons with sham group, $P<0.05$; " Comparisons with 1 day BS, $P<0.05$

\begin{tabular}{|c|c|c|c|c|c|}
\hline Group & 1 day $B S$ & $1^{\text {st }}$ day AS & $3^{\text {st }}$ day AS & $5^{\text {st }}$ day AS & $7^{\text {st }}$ day AS \\
\hline CCI model & $47.75 \pm 2.6$ & $27.00 \pm 1.69^{* \#}$ & $21.40 \pm 4.19^{* \#}$ & $17.9 \pm 2.51^{* \#}$ & $16.8 \pm 2.68^{* \#}$ \\
\hline Anti-VEGF & $48.20 \pm 0.91$ & $27.65 \pm 2.41^{* \#}$ & $32.9 \pm 1.91^{*} \Delta \#$ & $33.85 \pm 1.59 * \Delta \#$ & $29.3 \pm 3.54^{* \Delta \#}$ \\
\hline Rapamycin & $45.72 \pm 1.59$ & $29.00 \pm 2.10^{* \#}$ & $28.40 \pm 5.06^{* \Delta \#}$ & $25.8 \pm 2.21 * \Delta \#$ & $27.50 \pm 2.57^{* \Delta \#}$ \\
\hline DMSO control & $49.20 \pm 2.66$ & $25.85 \pm 1.38^{* \#}$ & $21.7 \pm 1.73^{* \#}$ & $19.45 \pm 1.84^{* \#}$ & $16.10 \pm 1.23^{* \#}$ \\
\hline NS control & $49.55 \pm 2.10$ & $27.90 \pm 2.20^{* \#}$ & $21.35 \pm 1.90^{* \#}$ & $17.15 \pm 2.03^{* \#}$ & $15.3 \pm 2.03^{* \#}$ \\
\hline Sham & $48.60 \pm 2.21$ & $47.55 \pm 0.97 \triangle$ & $47.48 \pm 0.65^{\Delta}$ & $46.45 \pm 1.40^{\Delta}$ & $47.75 \pm 1.40^{\Delta}$ \\
\hline Normal control & $46.30 \pm 1.12$ & $45.50 \pm 1.05^{\Delta}$ & $46.25 \pm 1.30^{\Delta}$ & $45.85 \pm 1.19 \Delta$ & $45.15 \pm 1.26^{\Delta}$ \\
\hline
\end{tabular}




\section{Cellular Physiology

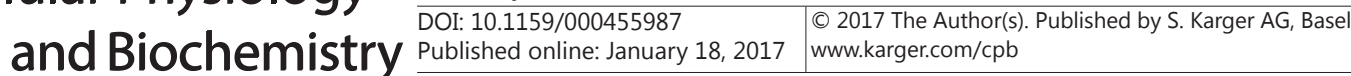 \\ Xie et al.: MicroRNA-183 Targets mTOR/VEGF Signaling Pathway in Neuropathic Pain}

Table 3. PWTL of rats' left posterior limbs ( $n=25$, mean \pm SD, unit: s). PWTL: paw withdrawal thermal latency; SD: standard deviation; BS: before surgery; AS: after surgery; CCI: chronic constrictive injury; DMSO: dimethylsulfoxide; VEGF: vascular endothelial growth factor; NS: normal saline. ${ }^{\triangle}$ Comparisons with CCI group, $P<0.05$; ${ }^{*}$ Comparisons with sham group, $P<0.05$; ${ }^{\#}$ Comparisons with 1 day BS, $P<0.05$

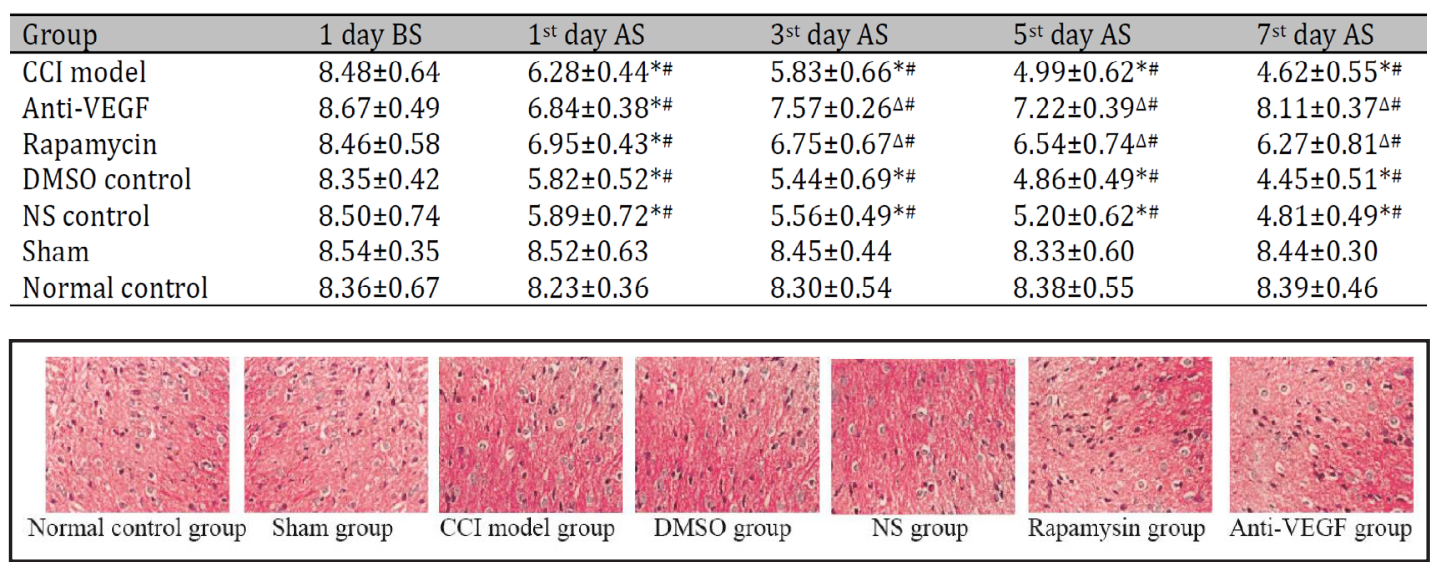

Fig. 2. HE (hermatoxylin eosin) staining of neuronal cells located in rats' lumbar spinal cord $(400 \times)$.

of PWMT were found after operations $(P<0.05)$. The rats' posterior limbs even exhibited claudication, mild ectropian, retraction of paw and inabilities to bear load at the $1^{\text {st }}$ day after surgery, whereas the above phenomena were scarcely detected before surgery. Obviously, the CCI, NS and DMSO group also differed from the control group in their post-operative PWMTs at each corresponding time point $(P<0.05)$ while there is no makeable difference among them $(P>0.05)$. Finally, the descending PWMT in rapamyclin or anti-VEGF group seemed apparently larger than CCI group but smaller than control group at the $3^{\text {rd }}, 5^{\text {th }}$ and $7^{\text {th }}$ day after surgery $(P<0.05)$, while no obvious discrepancies in PWMT were observed between rapamyclin and anti-VEGF group $(P>0.05)$. The rats' PWTL displayed the same tendencies with PWMT (Fig. 1B, Table 3).

\section{Morphology of neuronal cells located in rats' lumbar spinal cord}

Neuronal cells of all studied groups were neat in alignments, integral in morphology, clear in dying of cell nucleus and cytoplasm (Fig. 2). In addition, swelling, denaturation and necrosis of the cells were barely observed and no edema and infiltration of inflammatory cells within mesenchyme.

Expressions of miR-183, mTOR, GluR1 and GluR2 in superficial laminae of spinal dorsal horn

As shown in Figure 3A, the miR-183 expression in rapamyclin and VEGF antibody group was lower than the control group, but higher than CCI group $(P<0.05)$. While mTOR and VEGF expression in rapamyclin and VEGF antibody group was higher than the control group, but lower than CCI group. The number of mTOR positive cells of sham group remained very close to the control group, yet the positive cell numbers in the CCI, DMSO and NS groups were remarkably increased in comparison to the control group $(P<0.05)$. Similarly, the positive cell counts of rapamyclin or VEGF antibody group were larger than those of the control group, but smaller than those of CCI group $(P<0.05)$. Virtually, the variation trends of VEGF, GluR1 and GluR2 expressions were similar to those of mTOR expressions (Fig. 3B). In addition, as Figure 7 shown, the mTOR expressed mainly in the sensory neurons in superficial laminae of spinal dorsal horn, while VEGF, GluR1 and GluR2 expressed in both neurons and neuroglial cells. Moreover, the protein expression levels of mTOR, VEGF, GluR1 and GluR2 detected by western blot assay were also consistent with the changing conditions of positively expressional cell numbers (Fig. 3C-D). 


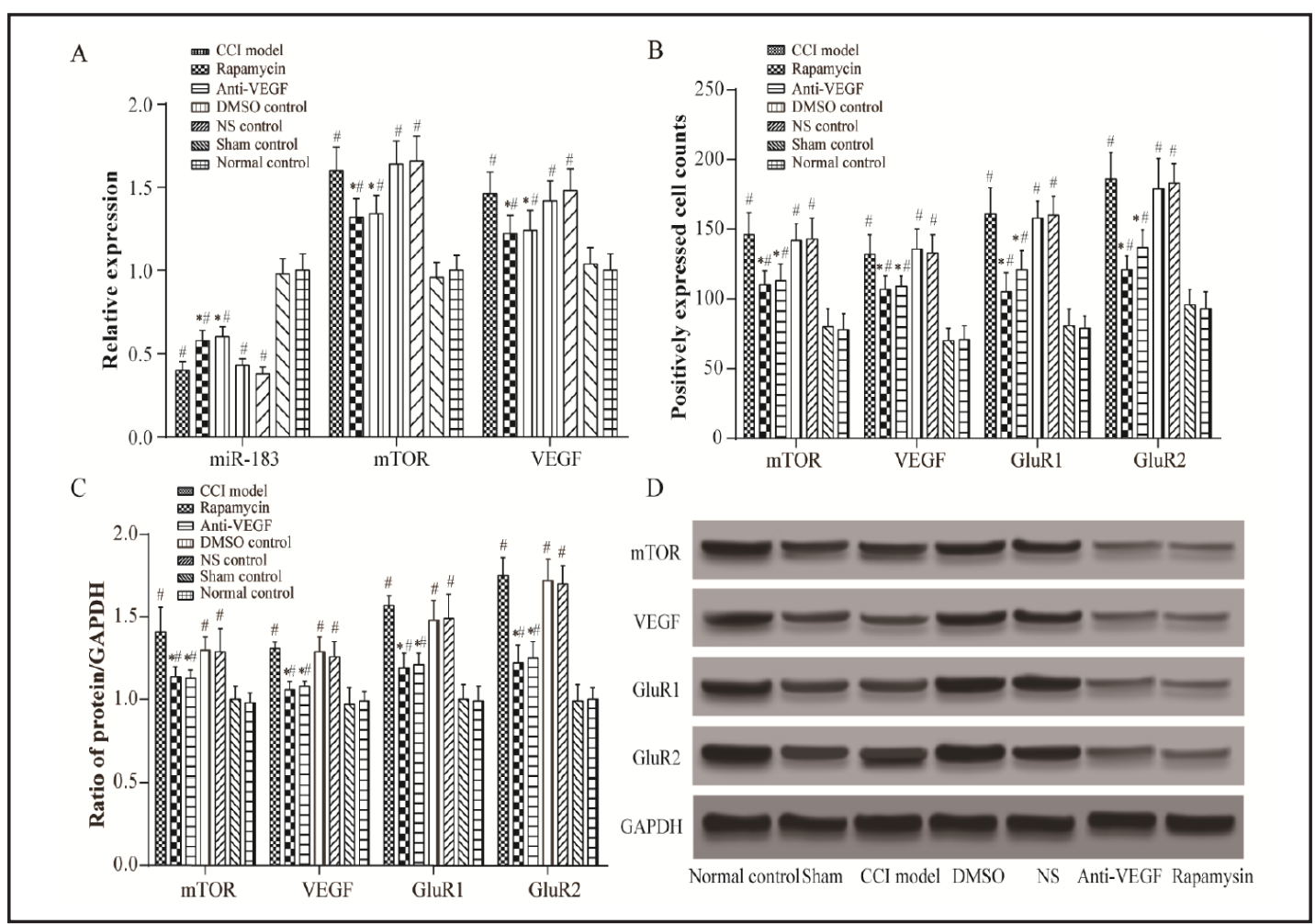

Fig. 3. Expressions of miR-183, mTOR, VEGF, GluR1 and GluR2 in rats' superficial laminae of spinal dorsal horn: (A) Relative expression of miR-183, mTOR and VEGF detected by qRT-PCR; (B) Positive cell numbers; (C) Ratio of protein/GAPDH; (D) Results detected by western blot analysis. ${ }^{*}$ Comparisons with CCI group, $P<0.05$; "Comparisons with the normal control group, $P<0.05$.

Regulation of mTOR and VEGF activity by direct binding of miR-183 to the 3'UTR of mTOR

On the basis of TargetScan, miR-183 was anticipated to target human mTOR 3'UTR at nucleotide position 902-925, with their mirSVR and PhastCons scores of -0.1115 and 0.7195 , respectively. The performance of dual luciferase reporter gene assays confirmed the direct binding of seed sequences of mature miR-183 to 3' UTR of mTOR mRNA (Fig. 4A). In addition, the expression of miR-183 was up-regulated in Pre-miR-183 subgroup and downregulated in LNA-miR-183 subgroup significantly $(P<0.05)$ in both mTOR-wt and mTORmut groups (Fig. 4B). Moreover, mTOR was over-expressed in Pre-miR-183 subgroup and down-regulated in LNA-miR-183 subgroup significantly $(P<0.05)$ in the mTOR-wt group, while no significant difference among groups in the mTOR-mut group with low expression (Fig. 4C). To be specific, loss of miR-183 in PC12 cells (LNA-miR-183 subgroup/LNA-Scr subgroup) advanced the luciferase activity $(P<0.05)$, while inhibited activity of luciferase was examined when miR-183 was over-expressed (Pre-miR-183 subgroup/Pre-miR-control subgroup) in PC12 cells with normally present mTOR $(P<0.05)$. Nonetheless, mutations of mTOR enabled the luciferase activity relatively stable in the above four groups, irrespective of miR-183 expression levels (all $P>0.05$ ) (Fig. 4D).

Effects of miR-183 on downstream mTOR and VEGF expressions

When the expression of miR-183 expression was upregulated, the protein levels of mTOR and VEGF were suppressed. However, once the miR-183 was blocked, the expression of mTOR and VEGF was ascending (Fig. 5).

Regulation of VEGF, GluR1 and GluR2 regulated by mTOR

With these PC12 cells, a series of western blotting assays were conducted to further determine whether VEGF was the downstream protein of mTOR. The results demonstrated 
Fig. 4. Direct regulation of mTOR with miR-183. (A) Putative binding sites for miR-183 in 3' UTR of mTOR; (B) The expression of miR-183; (C) The expression of mTOR; (D) Luciferase reporter assays of mTOR 3' UTR with modifying miR-183 (down-regulation: LNA-miR-183; up-regulation: PremiR-183) expressions. "Comparisons with the corresponding control group, $P<0.05$.
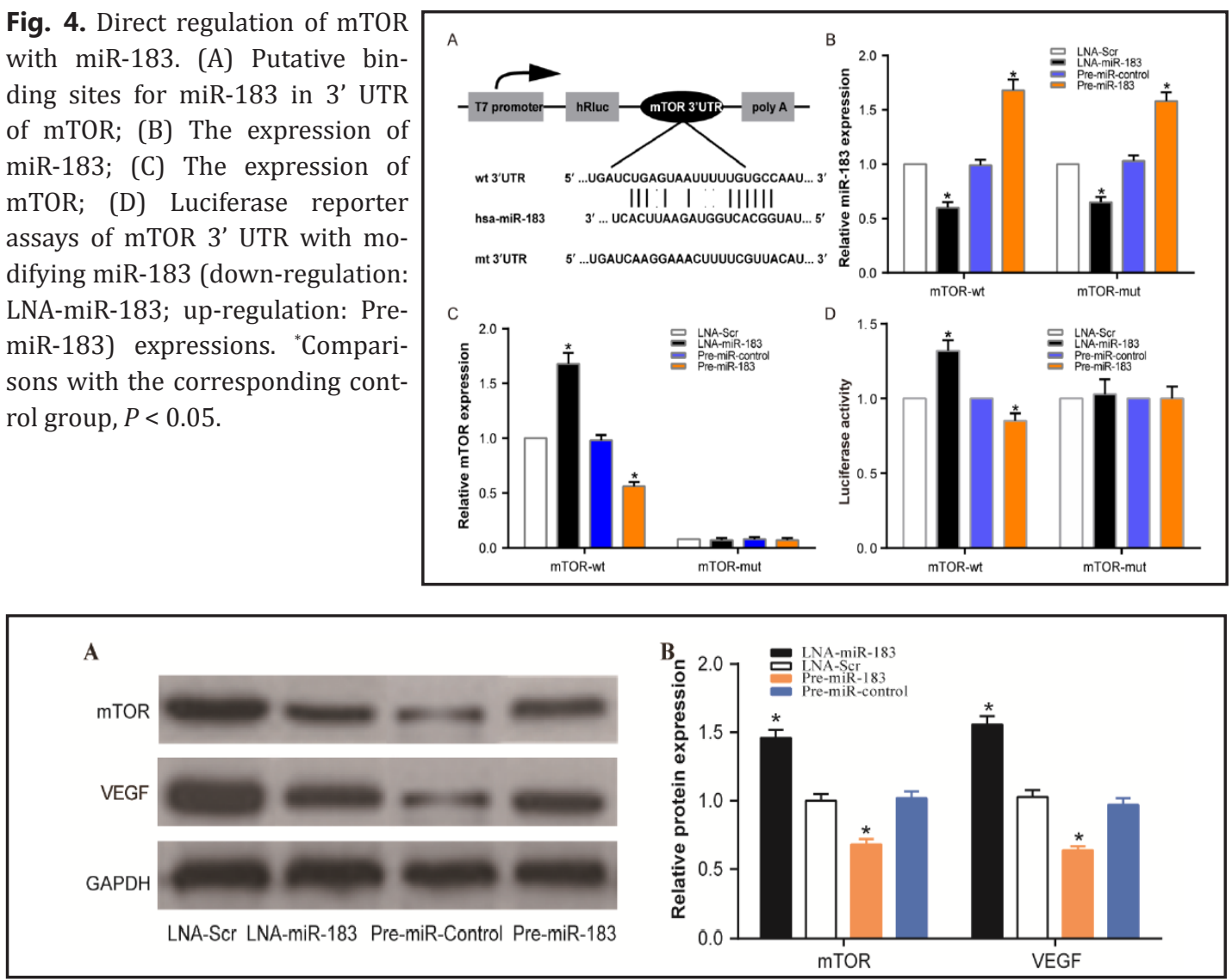

Fig. 5. Effects of modified miR-183 expressions on downstream mTOR and VEGF expressions. (A) Western-blot analysis showing that alteration of miR-183 expression changes protein levels of mTOR and VEGF; (B) Bar graphs representing quantification of mTOR and VEGF expressions normalized to GAPDH. *Comparisons with the corresponding control group, $P<0.05$.

that VEGF expressions were upregulated in the presence of increased mTOR expression $(P$ $<0.05$ ) (Fig. 6A-B). Nevertheless, mTOR expressions did not vary with expressional changes of VEGF $(P>0.05)$ (Fig. 6C-D), partly providing that mTOR was in the upstream of VEGF. Moreover, GluR1 and GluR2 were significantly upregulated when either mTOR or VEGF was over-expressed $(P<0.05)(P<0.05)$ (Fig. 6).

\section{Discussion}

Neuropathic pain caused by damages to nerve system offers no biological advantages but suffering and distress, which might result from infections, trauma, metabolic abnormalities, chemotherapy, surgery, irradiation, neurotoxins, inherited neurodegeneration, nerve compression, inflammation, and tumor infiltration [31]. Millions of people suffer from neuropathic pain, whereas its treatment and diagnosis are insufficient.

The present study demonstrated that elevated VEGF expression is significantly related to neuropathic pain, while the inhibition of VEGF helps to attenuate neuropathic pain. The result is in consistence with previous studies in diverse mouse models [18]. Considering its critical role in pain regulation, VEGF and VEGFR are regarded as valuable therapeutic targets. The anti-angiogenic VEGF-A splice variant, VEGF-A165b, was invented as a potential therapeutic for diabetic neuropathy. Results demonstrated that in animal models, VEGFA165b significantly altered pain behavior and prevented neuronal stress [32]. Hypoxia- 


\section{\begin{tabular}{ll} 
Cellular Physiology & Cell Physiol Biochem 2017;41:181-192 \\
\hline DOI: 10.1159/000455987 & 2017 The Author(s). Published by S. Karger AG, Basel
\end{tabular}

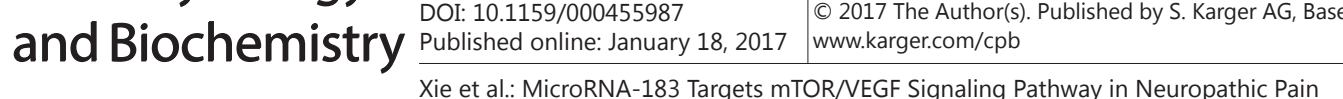

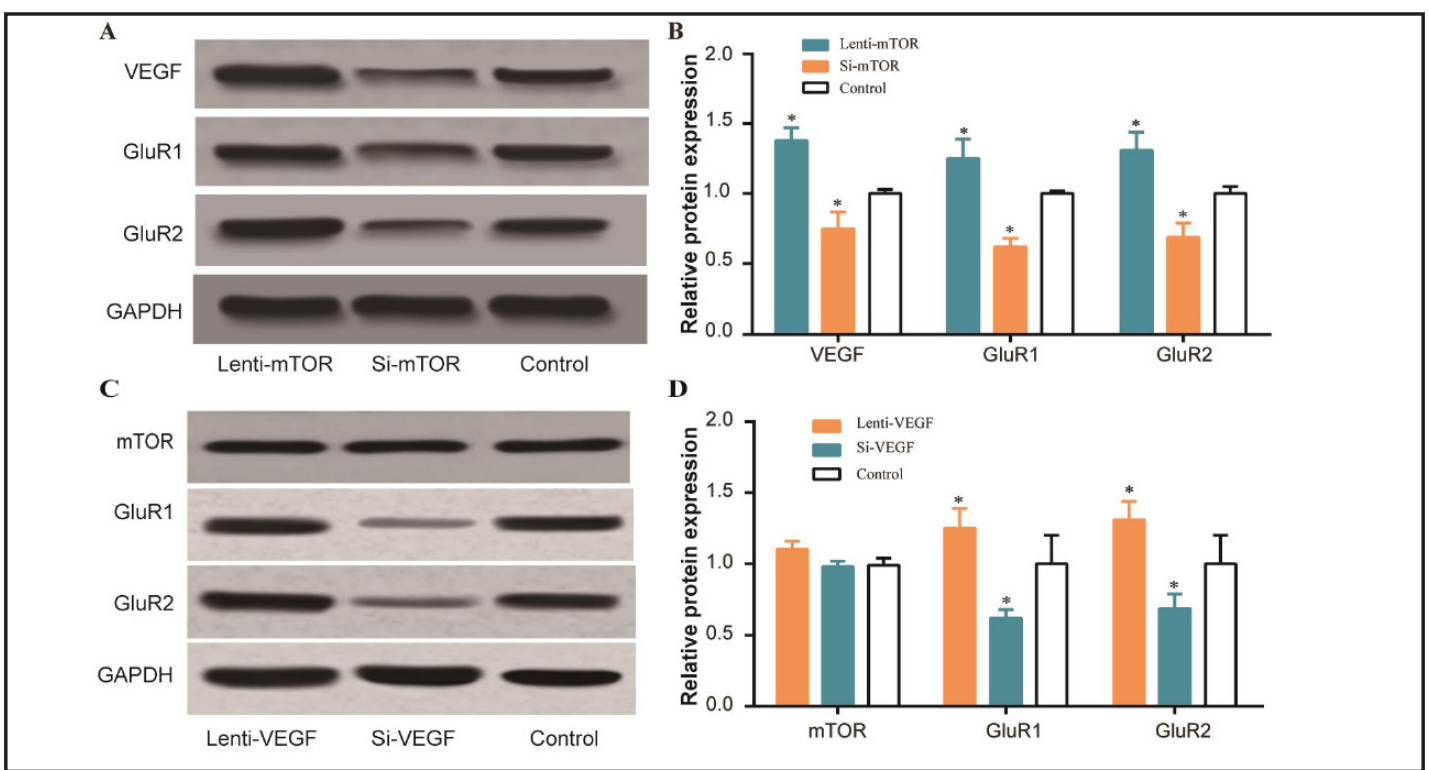

Fig. 6. Regulation of VEGF, GluR1 and GluR2 by mTOR (A) Western-blot analysis showing that altered mTOR expressions changes protein levels of VEGF, GluR1 and GluR2; (B) Bar graphs representing quantification of VEGF, GluR1 and GluR2 expressions normalized to GAPDH. (C) Western-blot analysis showing that altered VEGF expressions changes protein levels of GluR1 and GluR2, yet not those of mTOR; (D) Bar graphs representing quantification of mTOR, GluR1 and GluR2 expressions normalized to GAPDH. *Comparisons with control, $P<0.05$.

specific, VEGF-expressing neural stem cell therapy was also proposed as safe and effective treatment of neuropathic pain [33]. Previous results also showed that neural stem cells expressing VEGF aided functional recovery and pain reduction in a sciatic nerve injury model [34].

Zhang W et al. reported that intrathecal injection of rapamycin could reduce neuropathic pain by inhibiting mTOR [35]. Tateda $S$ et al. demonstrated that rapamycin, as an inhibitor of mTOR, suppressed the development of neuropathic pain when the spinal cord was injured [36]. These studies consisted with our observation that the inhibition of mTOR can significantly attenuate symptoms of neuropathic pain. Therefore, the inhibition of mTOR/ VEGF signaling pathway can relieve the neuropathic pain sufficiently.

Moreover, the miR-183 family is comprised of miR-96, miR-182 and miR-183, and they share sequence homology and play an important part in neuronal disorders. In addition to miR-183, miR-96 and miR-182 were shown to be able to reduce neuropathic pain as well. Chen et al. observed that miR-96 relieved neuropathic pain by inhibiting the expression of Nav1.3 in dorsal root ganglion [37]; whereas miR-182 inhibits Schwann cell proliferation and migration by targeting fibroblast growth factor 9 (FGF9) and neurotrimin (NTM) following sciatic nerve injury [38]. Interestingly, in addition to its positive role in pain regulation, increased miR-183 expression may also lead to neurodegenerative diseases. Elevated miR183 may reduce local axonal translation of mTOR in SMN (survival motor neuron)-deficient neurons and contribute to spinal muscular atrophy through targeting mTOR directly [28]. Our research illustrated that the mTOR was the direct target of miR-183 as well and miR-183 was negative regulator of both mTOR and VEGF.

In the CCI model of neuropathic pain, we also found that AMPA receptors located downstream of mTOR/VEGF signaling pathway. GluR1 and GluR2 were significantly upregulated when miR-183 was downregulated or when mTOR/VEGF was activated. In the pain system, glutamate is the primary excitatory transmitter. Two ionotropic glutamate receptors, AMPA and NMDA (N-methyl-D-aspartate), and the mGluRs (metabotropic G-protein coupled glutamate receptors) are involved in the transmission of peripheral pain signals [39]. 


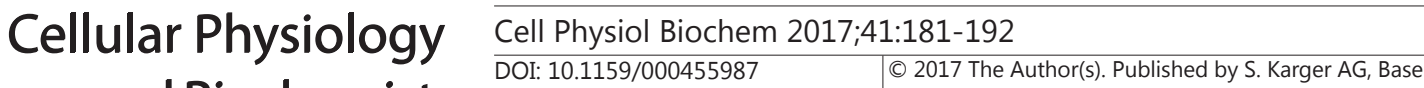

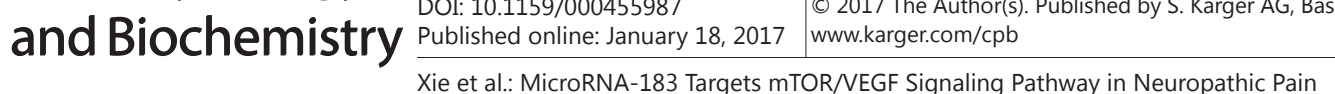
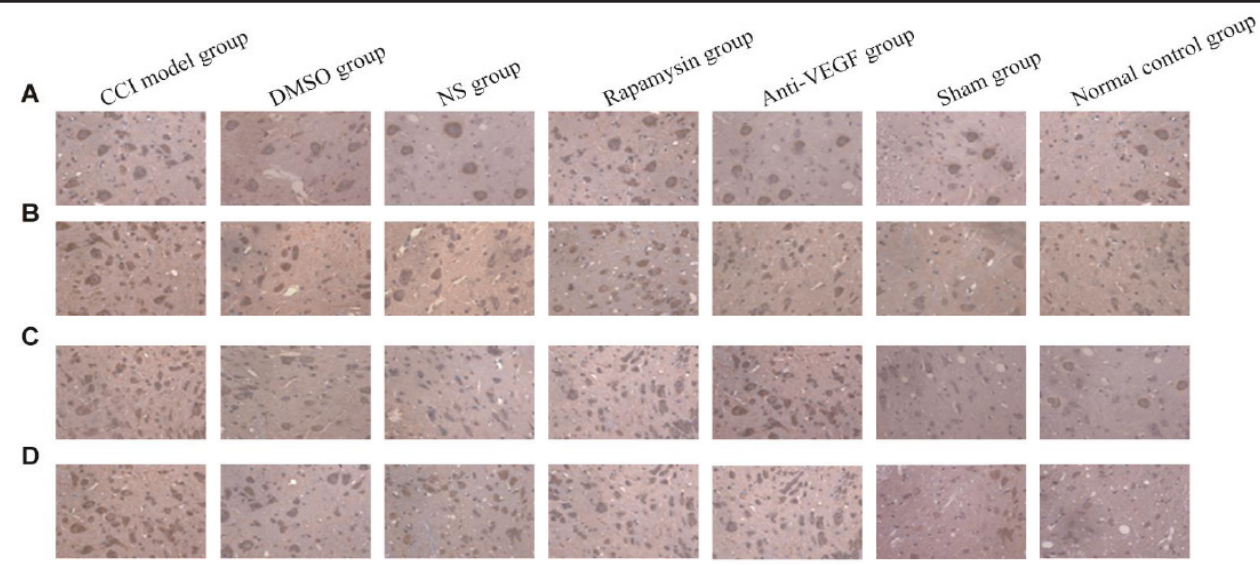

Fig. 7. Immunohistochemical staining of mTOR (A), VEGF (B), GluR1 (C) and GluR2 (D) under microscopic observation $(400 \times)$ in rats' superficial laminae of spinal dorsal horn.

Whereas the basic responses to pain stimuli is mediated by the activation of AMPA receptors in dorsal horn [40]. According to previous research, in CCI models, larger inward currents was induced in dorsal root ganglion by glutamate, selective ionotropic glutamate receptor agonists, and the group I metabotropic receptor (mGluR) agonist, but not NMDA agonists. Researchers also reported a 35\% increase in GluR1 and a 60\% decrease in GluR2 compared to uninjured controls in dorsal root ganglion in CCI model [25]. The contradictive results might be related to different location of sampling. In the process, excitatory aminoacids and neuropeptides are released by peripheral afferent fibers within the dorsal horn and results in postsynaptic changes of nociceptive neurons via the phosphorylation of NMDA and AMPA receptors [41]. Previous studies showed that GluR1 upregulation in the nucleus accumbens could help to minimize the depressive symptoms in chronic pain [42]. In addition to its peripheral role, AMPA receptors may play an important part in central nerve system during the sensory of pain as well. Therefore, further investigations need be conducted on the role of AMPA receptors in central nerve system concerning pain.

In summary, we manifested in the present study that the inhibition of mTOR/VEGF pathway can significantly attenuate symptoms of neuropathic pain. Besides, in the CCI model of neuropathic pain, the decrease of miR-183 could increase the expression of VEGF and AMPA receptors by upregulating mTOR directly. In addition, AMPA receptors, GluR1 and GluR2, located downstream of mTOR/VEGF signaling pathway.

\section{Disclosure Statement}

The authors declare no commercial or financial conflict of interest.

\section{References}

-1 Costigan M, Scholz J, Woolf CJ: Neuropathic pain: a maladaptive response of the nervous system to damage. Annu Rev Neurosci 2009;32:1-32.

-2 Bennett MI, Rayment C, Hjermstad M, Aass N, Caraceni A, Kaasa S: Prevalence and aetiology of neuropathic pain in cancer patients: a systematic review. Pain 2012;153:359-365.

-3 Bouhassira D, Lanteri-Minet M, Attal N, Laurent B, Touboul C: Prevalence of chronic pain with neuropathic characteristics in the general population. Pain 2008;136:380-387.

-4 D'Ammando A, Messina G, Franzini A, Dones I: Peripheral nerve field stimulation for chronic neuropathic pain: a single institution experience. Acta Neurochir (Wien) DOI:10.1007/s00701016-2713-8. 


\section{Cellular Physiology Cell Physiol Biochem 2017;41:181-192

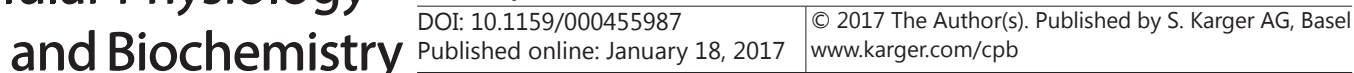 \\ Xie et al.: MicroRNA-183 Targets mTOR/VEGF Signaling Pathway in Neuropathic Pain}

-5 Ambros V: The functions of animal microRNAs. Nature 2004;431:350-355.

-6 Bartel DP: MicroRNAs: genomics, biogenesis, mechanism, and function. Cell 2004;116:281-297.

-7 Garcia-Larrea L, Peyron R: Pain matrices and neuropathic pain matrices: a review. Pain 2013;154:S29-43.

-8 Aldrich BT, Frakes EP, Kasuya J, Hammond DL, Kitamoto T: Changes in expression of sensory organ-specific microRNAs in rat dorsal root ganglia in association with mechanical hypersensitivity induced by spinal nerve ligation. Neuroscience 2009;164:711-723.

-9 Lin CR, Chen KH, Yang CH, Huang HW, Sheen-Chen SM: Intrathecal miR-183 delivery suppresses mechanical allodynia in mononeuropathic rats. Eur J Neurosci 2014;39:1682-1689.

10 Sciarretta S, Volpe M, Sadoshima J: Mammalian target of rapamycin signaling in cardiac physiology and disease. Circ Res 2014;114:549-564.

-11 Cetrullo S, D'Adamo S, Tantini B, Borzi RM, Flamigni F: mTOR, AMPK, and Sirt1: Key Players in Metabolic Stress Management. Crit Rev Eukaryot Gene Expr 2015;25:59-75.

-12 Asante CO, Wallace VC, Dickenson AH: Formalin-induced behavioural hypersensitivity and neuronal hyperexcitability are mediated by rapid protein synthesis at the spinal level. Mol Pain 2009;5:27.

13 Obara I, Tochiki KK, Geranton SM, Carr FB, Lumb BM, Liu Q, Hunt SP: Systemic inhibition of the mammalian target of rapamycin (mTOR) pathway reduces neuropathic pain in mice. Pain 2011;152:2582-2595.

14 Riaz SK, Iqbal Y, Malik MF: Diagnostic and therapeutic implications of the vascular endothelial growth factor family in cancer. Asian Pac J Cancer Prev 2015;16:1677-1682.

-15 Bahnassy A, Mohanad M, Ismail MF, Shaarawy S, El-Bastawisy A, Zekri AR: Molecular biomarkers for prediction of response to treatment and survival in triple negative breast cancer patients from Egypt. Exp Mol Pathol 2015;99:303-311.

-16 Blaha V, Stasek J, Bis J, Fortunato J, Andrys C, Pavlik V, Polansky P, Brtko M, Sobotka L: The role of VEGF in the diabetic patients undergoing endovascular therapy of symptomatic aortic valve stenosis. Physiol Res 2014;63:S351-359.

17 Wang J, Chen S, Jiang F, You C, Mao C, Yu J, Han J, Zhang Z, Yan H: Vitreous and plasma VEGF levels as predictive factors in the progression of proliferative diabetic retinopathy after vitrectomy. PLoS One 2014;9:e110531.

-18 Selvaraj D, Gangadharan V, Michalski CW, Kurejova M, Stosser S, Srivastava K, Schweizerhof M, Waltenberger J, Ferrara N, Heppenstall P, Shibuya M, Augustin HG, Kuner R: A Functional Role for VEGFR1 Expressed in Peripheral Sensory Neurons in Cancer Pain. Cancer Cell 2015;27:780796.

19 Brugarolas J, Kaelin WG Jr: Dysregulation of HIF and VEGF is a unifying feature of the familial hamartoma syndromes. Cancer Cell 2004;6:7-10.

-20 Wan X, Shen N, Mendoza A, Khanna C, Helman LJ: CCI-779 inhibits rhabdomyosarcoma xenograft growth by an antiangiogenic mechanism linked to the targeting of mTOR/Hif-1alpha/ VEGF signaling. Neoplasia 2006;8:394-401.

-21 Argyriou P, Papageorgiou SG, Panteleon V, Psyrri A, Bakou V, Pappa V, Spathis A, Economopoulou P, Papageorgiou E, Economopoulos T, Rontogianni D: Hypoxia-inducible factors in mantle cell lymphoma: implication for an activated mTORC1-->HIF-1alpha pathway. Ann Hematol 2011;90:315-322.

-22 Dingledine R, Borges K, Bowie D, Traynelis SF: The glutamate receptor ion channels. Pharmacol Rev 1999;51:7-61.

-23 Greger IH, Ziff EB, Penn AC: Molecular determinants of AMPA receptor subunit assembly. Trends Neurosci 2007;30:407-416.

-24 Liu TY, Cheng Y, Qin XY, Yu LC: Pharmacologically inhibiting GluR2 internalization alleviates neuropathic pain. Neurosci Bull 2015;31:611-616.

-25 Gong K, Kung LH, Magni G, Bhargava A, Jasmin L: Increased response to glutamate in small diameter dorsal root ganglion neurons after sciatic nerve injury. PLoS One 2014;9:e95491.

-26 Takei N, Inamura N, Kawamura M, Namba H, Hara K, Yonezawa K, Nawa H: Brain-derived neurotrophic factor induces mammalian target of rapamycin-dependent local activation of translation machinery and protein synthesis in neuronal dendrites. J Neurosci 2004;24:97609769 . 


\section{Cellular Physiology Cell Physiol Biochem 2017;41:181-192

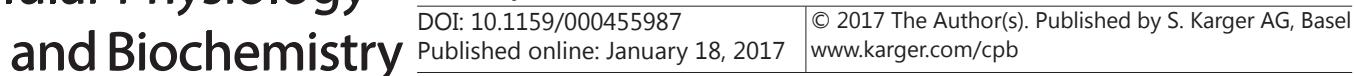 \\ Xie et al.: MicroRNA-183 Targets mTOR/VEGF Signaling Pathway in Neuropathic Pain}

-27 Bennett GJ, Xie YK: A peripheral mononeuropathy in rat that produces disorders of pain sensation like those seen in man. Pain 1988;33:87-107.

-28 Kye MJ, Niederst ED, Wertz MH, Goncalves Ido C, Akten B, Dover KZ, Peters M, Riessland M, Neveu P, Wirth B, Kosik KS, Sardi SP, Monani UR, Passini MA, Sahin M: SMN regulates axonal local translation via miR-183/mTOR pathway. Hum Mol Genet 2014;23:6318-6331.

29 Di Nardo A, Kramvis I, Cho N, Sadowski A, Meikle L, Kwiatkowski DJ, Sahin M: Tuberous sclerosis complex activity is required to control neuronal stress responses in an mTORdependent manner. J Neurosci 2009;29:5926-5937.

-30 Mou L, Zhang Q, Wang Y, Zhang Q, Sun L, Li C, Huang W, Yuan Y, Duan Y, Diao R, Jiang Z, Ye J, Cai $\mathrm{Z}$, Gui Y: Identification of Ube $2 \mathrm{~b}$ as a novel target of androgen receptor in mouse sertoli cells. Biol Reprod 2013;89:32.

- 31 Dworkin RH, Backonja M, Rowbotham MC, Allen RR, Argoff CR, Bennett GJ, Bushnell MC, Farrar JT, Galer BS, Haythornthwaite JA, Hewitt DJ, Loeser JD, Max MB, Saltarelli M, Schmader KE, Stein C, Thompson D, Turk DC, Wallace MS, Watkins LR, Weinstein SM: Advances in neuropathic pain: diagnosis, mechanisms, and treatment recommendations. Arch Neurol 2003;60:1524-1534.

-32 Hulse RP, Beazley-Long N, Ved N, Bestall SM, Riaz H, Singhal P, Ballmer Hofer K, Harper SJ, Bates DO, Donaldson LF: Vascular endothelial growth factor-A165b prevents diabetic neuropathic pain and sensory neuronal degeneration. Clin Sci (Lond) 2015;129:741-756.

-33 Lee HL, Lee HY, Yun Y, Oh J, Che L, Lee M, Ha Y: Hypoxia-specific, VEGF-expressing neural stem cell therapy for safe and effective treatment of neuropathic pain. J Control Release 2016;226:2134.

-34 Lee HL, Oh J, Yun Y, Lee HY, You Y, Che L, Lee M, Kim KN, Ha Y: Vascular endothelial growth factor-expressing neural stem cell for the treatment of neuropathic pain. Neuroreport 2015;26:399-404.

-35 Zhang W, Sun X-F, Bo J-H, Zhang J, Liu X-J, Wu L-P, Ma Z-L, Gu X-P: Activation of mTOR in the spinal cord is required for pain hypersensitivity induced by chronic constriction injury in mice. Pharmacol Biochem Behav 2013;111:64-70.

- 36 Tateda S, Kanno H, Ozawa H, Sekiguchi A, Yahata K, Yamaya S, Itoi E: Rapamycin suppresses microglial activation and reduces the development of neuropathic pain after spinal cord injury. J Orthop Res DOI:10.1002/jor.23328.

-37 Chen HP, Zhou W, Kang LM, Yan H, Zhang L, Xu BH, Cai WH: Intrathecal miR-96 inhibits Nav1.3 expression and alleviates neuropathic pain in rat following chronic construction injury. Neurochem Res 2014;39:76-83.

-38 Yu B, Qian T, Wang Y, Zhou S, Ding G, Ding F, Gu X: miR-182 inhibits Schwann cell proliferation and migration by targeting FGF9 and NTM, respectively at an early stage following sciatic nerve injury. Nucleic Acids Res 2012;40:10356-10365.

39 Bleakman D, Alt A, Nisenbaum ES: Glutamate receptors and pain. Semin Cell Dev Biol 2006;17:592-604.

40 Nickel FT, Seifert F, Lanz S, Maihöfner C: Mechanisms of neuropathic pain. Eur Neuropsychopharmacol 2012;22:81-91.

-41 Baron R, Binder A, Wasner G: Neuropathic pain: diagnosis, pathophysiological mechanisms, and treatment. Lancet Neurol 2010;9:807-819.

42 Su C, D'Amour J, Lee M, Lin HY, Manders T, Xu D, Eberle SE, Goffer Y, Zou AH, Rahman M, Ziff E, Froemke RC, Huang D, Wang J: Persistent pain alters AMPA receptor subunit levels in the nucleus accumbens. Mol Brain 2015;8:46. 